\title{
Submacroscopically Stable Equilibria OF BODIES UNDERGOING DisARRANGEMENTS AND DISSIPATION
}

\author{
Luca Deseri \\ Department of Mechanical and Structural Engineering \\ Università di Trento \\ 38050 Trento, ITALY \\ David R. Owen \\ Department of Mathematical Sciences \\ Carnegie Mellon University \\ Pittsburgh, PA 15213, USA \\ (Corresponding author. Tel: +1-412-268-8481; fax +1-412-268-6380. \\ E-mail address: do04@andrew.cmu.edu)
}

July 7, 2008

\begin{abstract}
Elasticity is viewed here as a starting point in the description of inelastic behavior. The two-scale geometry provided by structured deformations and a field theory of elastic bodies undergoing disarrangements (non-smooth geometrical changes) and dissipation are used to formulate and illustrate a concept of "submacroscopically stable configuration." A body in a submacroscopically stable equilibrium configuration resists additional submacroscopic geometrical changes such as the occurrence of microslips, the formation of microvoids, and the appearance of localized distortions that, together, leave the macroscopic configuration of the body unchanged. Submacroscopically stable configurations represent energetically preferred phases for bodies in equilibrium, and a procedure is described here for determining the submacroscopically stable equilibria of a body. The procedure is carried out in detail here for two classes of bodies that may undergo disarrangements and experience internal dissipation. One class is characterized by its bi-quadratic free energy response function, and the requirement of submacroscopic stability reduces from five to one the number of phases available to a body that is in equilibrium under mixed boundary conditions. For a subclass of these bi-quadratic free energy functions, the distinguished phase determined through this procedure has the same free energy response as one derived for nematic elastomers via statistical calculations. Boundary-value problems for the
\end{abstract}


macroscopic deformation corresponding to a submacroscopically stable equilibrium in a body of this class are formulated. A second class of bodies, the "near-sighted fluids," has both a prolate and a spherical phase that may occur in equilibrium; the submacroscopically stable equilibria of a near-sighted fluid must be stress-free, without regard to the particular phase that appears. In all considerations in this article, the term "equilibrium" is synonymous with satisfaction of balance of forces and moments in a given environment.

Keywords: C:stability and bifurcation, C:energy methods, A: phase transformation, A: microstructures

\section{Contents}

1 Introduction $\quad 3$

2 Background material $\quad 7$

2.1 Structured deformations . . . . . . . . . . . . . . . . 7

2.2 Field relations in statics . . . . . . . . . . . . . 7

3 Submacroscopic stability 9

3.1 Equilibrium structured configurations . . . . . . . . . . . . . 9

3.2 The augmented energy . . . . . . . . . . . . . . . . . . 10

3.3 Submacroscopically stable equilibria . . . . . . . . . . . . . 12

3.4 A procedure for determining submacroscopically stable equilibria 15

4 Example: biquadratic energy $\Psi_{K O} \quad \mathbf{1 6}$

4.1 Portfolio of phases . . . . . . . . . . . . . 16

4.1.1 Solutions of the consistency relations . . . . . . . . . 16

4.1.2 Submacroscopic geometry of the phases . . . . . . . . 18

4.1.3 The augmented energy in each $\Psi_{K O}$-phase . . . . . . . 19

4.2 Pure-phase energy minimization within each

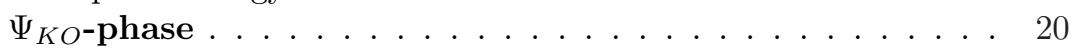

4.2.1 Classical, spherical, and textured phases . . . . . . . . 20

4.2.2 Prolate and oblate phases . . . . . . . . . . . . . 21

4.3 Pure-phase energy minimization among the

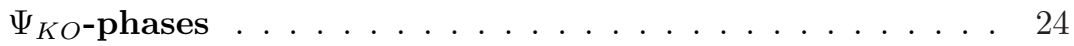

4.4 Boundary value problems for the determination of submacroscopically stable equilibria . . . . . . . . . . . . . 25

5 Example: near-sighted fluids $\quad 26$

6 Conclusions and outlook $\quad 28$ 


\section{Introduction}

The modelling of inelastic behavior in materials typically rests on the identification of mechanisms through which a given material deviates from elastic behavior. These mechanisms often are associated with particular changes in the geometry of a body and with accompanying internal dissipation. In phenomenological models such as elastoplasticity, viscoplasticity, and viscoelasticity, the deviation from elastic behavior is modelled through the introduction of additional variables in constitutive relations, such as plastic deformation, rates of macroscopic deformation, or histories of deformation. Refinements of these models that describe specific materials are obtained by linking the additional variables directly or indirectly to submacroscopic phenomena such as crystallographic slip, formation of voids, movement of vacancies, and phase transitions. These phenomena represent geometrical mechanisms for disrupting the underlying smooth distortion of a lattice or of a polymer network that, by themselves, lead to purely elastic behavior. From this point of view, elasticity may be regarded as a starting point in the description of inelastic behavior.

The purposes of this paper are (1) the introduction of a notion of stability of equilibria of bodies against the purely submacroscopic geometrical changes associated with inelastic behavior, and (2) the illustration of this notion through two examples. The need for a concept of "submacroscopically stable equilibria" arises from the fact that, by itself, the condition that an elastic body satisfy equations of balance of forces and moments in a given environment does not generally single out a preferred phase in which the body may appear. The notion of submacroscopic stability introduced and studied here is presented in the context of elastic bodies undergoing disarrangements and dissipation (Deseri and Owen, 2003). This field theory is a generalization of dynamical, non-linear elasticity that is formulated in the geometrical setting of structured deformations (Del Piero and Owen, 1993). This setting affords a precise distinction between classical, macroscopic changes of the geometrical configuration of a body, on the one hand, and purely submacroscopic geometrical changes, on the other hand. In connection with crystalline plasticity, structured deformations have been employed to derive basic kinematical relations usually assumed in treatments of multiple slip in single crystals (Deseri and Owen, 2002b) and to relate hardening properties of single crystals to submacroscopic instabilities and to the separation of active slip bands in crystals (Deseri and Owen, 2000, 2002a). In this context, a single crystal in a submacroscopically stable equilibrium configuration would resist crystallographic slips and lattic distortions that leave the macroscopic configuration of the crystal unchanged.

At the macroscopic and submacroscopic levels, the class of structured deformations is sufficiently broad to permit both smooth geometrical changes and non-smooth geometrical changes (disarrangements) to occur. Here, as in [?], we restrict our attention to the case where only smooth macroscopic geometrical changes occur, thereby placing emphasis on the role that submacroscopic disarrangements can play in the description of inelastic behavior. In this context, a structured deformation $(g, G)$ is a pair in which $g$ is the macroscopic 
deformation field and $G$ is a tensor field called the deformation without disarrangements. Each structured deformation $(g, G)$ can be factored as a composition of a classical deformation, $(g, \nabla g)$ that causes no disarrangements, and a purely submacroscopic deformation, $(i, K)$ that causes no macroscopic geometrical changes. Moreover, the latter deformation allows the body to undergo both smooth and non-smooth submacroscopic changes, such as smooth deformations of a lattice and non-smooth slips or incorporation of voids. From a different point of view the purely submacroscopic factor $(i, K)$ in the factorization describes the deformation that the body undergoes starting from a virgin configuration and ending in the reference configuration, while the classical factor $(g, \nabla g)$ takes the body from the reference configuration into the final, deformed configuration. Macroscopically, the virgin and reference configurations are identical, because $i$ is the identity mapping, while, when $K \neq \nabla i$, the reference configuration differs submacroscopically from the virgin configuration and displays the phase, or mixture of phases, associated with the body in that configuration. In contrast to this, the reference and final deformed configurations differ macroscopically, in general, while they do not differ submacroscopically, because the classical factor $(g, \nabla g)$ introduces no disarrangements in addition to ones caused by $(i, K)$.

The field relations derived in Deseri and Owen, 2003, for elasticity with disarrangements and dissipation provide a coupling between the fields $g$ and $K$ and predict the spectrum of phases that is available to a body, both in statical and dynamical settings, once the free energy response function of the body is known. For a given macroscopic configuration $g$ of the body, there typically are at least two phases $K$ compatible with the field relations, as illustrated in the examples presented here. Given $g$, the notion of stability that we study here then provides a means of selecting among the available phases $K$ for that configuration the one (or, possibly, more than one) that is "energetically preferred."

According to the field theory of Deseri and Owen, 2003, elastic bodies undergoing disarrangements may and typically do experience internal dissipation while undergoing smooth macroscopic deformations. In this broader field theory, the dissipation inequality in the isothermal case is the assertion that the stress power $S \cdot \nabla g^{\prime}$ is no less than the rate of change $\psi^{\prime}$ of the density $\psi$ of free energy and appears as a non-trivial field relation, while in classical non-linear elasticity the dissipation inequality is identically satisfied as an equality. We show in Section 3 that the dissipation inequality and an appropriate choice of variables $F=\nabla g$ and $\Delta=\nabla g(2 K-I)$ lead directly to an "augmented energy" $\mathcal{I}(g, \Delta)$ with the following properties:

1. for a given field $\Delta$ measuring the disparity between deformations due to and without disarrangements, the variation of the augmented energy $\mathcal{I}(g, \Delta)$ with respect to macroscopic configuration $g$ vanishes if and only if the body is in equilibrium in a given environment, i.e., if and only if the equations of balance of forces and moments hold throughout the body in that environment;

2. the rate of change $\frac{d}{d \tau} \mathcal{I}\left(g_{\tau}, \Delta_{\tau}\right)$ of the augmented energy of the body on 
processes $\tau \mapsto\left(g_{\tau}, \Delta_{\tau}\right)$ that pass smoothly through equilibrium configurations (quasistatic processes) within a time-independent environment is equal to the rate of increase $\int_{B} \psi^{\prime}$ of the free energy of the body minus the rate at which the body dissipates energy, $\int_{B}\left(S \cdot \nabla g^{\prime}-\psi^{\prime}\right)$.

We emphasize that "equilibrium" here, by definition, means that balance of forces and moments holds, while, in some approaches to statics, "equilibrium" is defined by means of a stationarity or extremality condition for an energy functional. Consequently, the first statement tells us that the condition that a body be in equilibrium in a given environment is characterized by a stationarity property of the augmented energy. The second statement and the definition of the augmented energy imply that during purely submacroscopic quasistatic processes in a time-independent environment, the augmented energy cannot increase and, hence, the body stores energy no more rapidly than it dissipates energy.

These two results form the basis not only for the definition of submacroscopically stable equilibria that we give in Section 3 but also for the two-step procedure that we describe in that section for determining such equilibria. In the first step, one determines, for a given macroscopic deformation $g$ that satisfies prescribed placement boundary conditions, the disparity field(s) $\Delta$ that minimize the augmented energy $\mathcal{I}(g, \Delta)$, subject to satisfaction of all of the underlying field relations for an elastic body undergoing disarrangements and dissipation, except for the balance of forces and the traction boundary conditions. This step selects the energetically preferred phases of the body for the given macroscopic configuration $g$.

In the second step, a minimizing disparity field $\Delta_{s s}$ is chosen for the given macroscopic field $g$ and both are substituted into the balance of forces and traction boundary condition, yielding a boundary value problem for the determination of the macroscopic configuration $g$ as well as for the possible determination of any parameters in the description of $\Delta_{s s}$ that are not already determined by $g$. A solution $g$ of this boundary value problem that is both injective and orientation-preserving provides a submacroscopically stable equilibrium configuration $\left(g, \Delta_{s s}\right)$ of the body. In cases where there is exactly one preferred phase $\Delta_{s s}$ for the given macroscopic configuration $g$, the boundary value problem can be regarded as one for the non-linearly, Cauchy elastic body whose response is obtained by restricting the response functions of the original elastic body undergoing disarrangements to the energetically preferred phase $\Delta_{s s}$, so that the stress and free energy can be expressed as functions of $\nabla g$, alone.

In Sections 4 and 5 we carry out the first step of this procedure completely for each of two choices of the free energy response function of the body. In Section 4, the first-step minimization selects, from a portfolio of five phases admitted by the field relations, exactly one of these phases as energetically preferred - the spherical phase, the classical phase, or the prolate phase, depending upon the value of the ratio of the two material constants that appear as coefficients in the biquadratic expression for the free energy. In Section 5, 
the first-step minimization procedure does not select between the spherical and prolate phases available to the body, but rather selects only stress-free configurations as candidates for energetically preferred submacroscopic geometrical states. For these "near-sighted" fluids, the selection of stress-free configurations amounts to choosing values of the volume fraction $\operatorname{det} K$ that minimize the free energy $\psi(\operatorname{det} K)$ and make its derivative zero.

The second step in the determination of submacroscopically stable equilibrium is treated in Section 4.4 for bodies with the biquadratic energy introduced at the beginning of Section 4. For each of the three phases identified in the first step, the corresponding boundary value problem is recorded and briefly discussed. For the nearsighted elastic bodies studied in Section 5, the second step is carried out completely, and a characterization of submacroscopically stable equilibria is obtained.

The notion of submacroscopic stability and the associated minimization problem analyzed here are formulated in the context of elasticity with disarrangements and dissipation (Deseri and Owen, 2003). However, in the context of statistical models of nematic elastomers (Bladon et al, 1993), the appearance of a director field $d$ as well as the macroscopic deformation gradient $\nabla g$ in the formula for the free energy density is treated in (Bladon et al, 1993) in a manner similar to the first step in the present procedure: for a given value of the deformation gradient, the director $d$ is chosen in Bladon et al, 1993 so that the free energy density is minimized. That minimization corresponds to the firststep minimization carried out in Section 4 for the case of prolate, oblate, and textured phases. Moreover, the competing prolate phase discussed in Section 4 provides a formula for the free energy density here that has a form close to the energy derived in Bladon et al, 1993 from statistical considerations. In our opinion, the concepts and procedure introduced here provide a framework that more fully justifies treatments, such as that of the director $d$ in Bladon et al, 1993, in which energy minimization is used to select preferred values for fields that reflect submacroscopic geometrical changes.

It is worth emphasizing that submacroscopically stable equilibria in the present context need not be macroscopically stable. The additional requirement of macroscopic stability, when precisely formulated, may necessitate the consideration of additional geometrical complexity beyond the selection of phases that emerges in the present treatment of submacrosocopic stability. This possibility is suggested in the analysis of Silhavy, 2004, and of DeSimone and Doltzmann, 2002, of relaxed energies for nematic elastomers based on the energy derived in Bladon et al, 1993. Although the minimization in Bladon et al, 1993 that links the director $d$ to the macroscopic deformation gradient $\nabla g$ places a strong constraint on the submacroscopic state of the body, the subsequent relaxation of the resulting energy density carried out in Silhavy, 2004, and in DeSimone and Doltzmann, 2002, provides via minimizing sequences $n \mapsto \nabla g_{n}$ additional geometrical structure to candidates for absolutely stable configurations. 


\section{Background material}

\subsection{Structured deformations}

For present purposes, a structured deformation is a pair $(g, G)$ with $g$ the smooth (i.e. continuously differentiable) deformation of a given body $\mathcal{B}$ and $G$ a continuous tensor field on $\mathcal{B}$ called the deformation without disarrangements. $G$ represents the part of the macroscopic deformation gradient $\nabla g$ due to smooth, submacroscopic geometrical changes, and the inequality

$$
0<m<\operatorname{det} G(X) \leq \operatorname{det} \nabla g(X) \text { for all } x \in \mathcal{B}
$$

guarantees that no interpenetration of matter occurs submacroscopically (Del Piero and Owen, 1993). The tensor field $M:=\nabla g-G$ is called the deformation due to disarrangements, because the values $M(X)$ of the field $M$ can be shown to represent a limit of averages of jumps of injective, piecewise smooth deformations that approximate $g$ and whose gradients approximate $G$ (Del Piero and Owen, 1993),(Del Piero and Owen, 1995). Thus, each structured deformation $(g, G)$ provides an additive decomposition

$$
\nabla g=G+M
$$

of the macroscopic deformation gradient that identifies the contributions to $\nabla g$ of both smooth and non-smooth submacroscopic geometrical changes. Since $G$ is invertible at each point of the body, the additive decomposition of $\nabla g$ yields a multiplicative decomposition $\nabla g=G\left(I+G^{-1} M\right)$, with $I$ the identity tensor field, as well as the multiplicative relation

$$
G=\nabla g K
$$

with $K:=(\nabla g)^{-1} G=\left(I+G^{-1} M\right)^{-1}$. The tensor field $K$ determines the structured deformation $(i, K)$, where $i$ denotes the identity mapping on the body. This deformation takes the body in a given "virgin configuration" and introduces only submacroscopic geometrical changes, bringing the body into its reference configuration from which the body can undergo the classical deformation $(g, \nabla g)$. The inequality (1) tells us that the tensor field $K$ satisfies

$$
0<\operatorname{det} K \leq 1
$$

\subsection{Field relations in statics}

The field relations for a moving elastic body undergoing disarrangements and dissipation that were introduced by Deseri and Owen, 2003, take the following form in statics, i.e., when all fields are independent of time:

$$
\begin{gathered}
\operatorname{div}\left(D_{G} \Psi+D_{M} \Psi\right)+b_{\text {ref }}=0 \\
\left(D_{G} \Psi\right) M^{T}+\left(D_{M} \Psi\right)(G+M)^{T}=0
\end{gathered}
$$




$$
\begin{gathered}
\operatorname{sk}\left[\left(D_{G} \Psi\right) M^{T}+\left(D_{M} \Psi\right) G^{T}\right]=0 \\
\operatorname{det} G \leq \operatorname{det}(G+M) \\
F:=\nabla g=G+M .
\end{gathered}
$$

Here, the response function $(G, M) \mapsto \Psi(G, M)$ for the given elastic body provides at each point $X$ the Helmholtz free energy $\psi(X)=\Psi(G(X), M(X)$ ) (measured per unit volume in the reference configuration) associated with a given structured deformation $(g, G)$, and $D_{G} \Psi$ and $D_{M} \Psi$ denote the partial derivatives of the free energy response function. The "consistency relation" in the form (6) arises from an additive decomposition for the density of contact forces into parts with and without disarrangements, as well as from the specific constitutive relations that connect stresses with and without disarrangements to the response function $\Psi$ (Deseri and Owen, 2003). The relation (7) asserts that the skew part of the field $\left(D_{G} \Psi\right) M^{T}+\left(D_{M} \Psi\right) G^{T}$ vanishes at every point of the body and arises from the requirement that the internal dissipation be frameindifferent. In addition to the additive decomposition of deformation gradient (2) that rests on geometrical considerations alone, an additive decomposition of the Piola-Kirchhoff stress field $S$ results from the constitutive assumptions that define the elastic body under consideration (Deseri and Owen, 2003),

$$
S=D_{G} \Psi+D_{M} \Psi
$$

and it follows that the equation (5) expresses the balance of forces on the body. In that equation, $b_{r e f}$ denotes the body force field, measured per unit volume in the reference configuration. Frame-indifference of the response function $\Psi$ and frame-indifference of the internal dissipation (7) imply (Deseri and Owen, 2003) that the Cauchy stress $T \circ g=(\operatorname{det} \nabla g)^{-1} S(\nabla g)^{T}$ is symmetric, so that balance of moments holds throughout the body.

The additive decomposition (2), the consistency relation (6), and the frameindifference relation (7) imply that the above field relations for elastostatics with disarrangements amount to twelve scalar equations restricting the three scalar components of $g$ and nine components of $G$. We note that the constitutive relations for the body require that the decomposition of the stress (10) hold at each point in the body.

For the notion of equilibrium configuration defined in Section 3, the field relations above are best recast in terms of the macroscopic deformation $g$ and the "disparity" $\Delta:=G-M$, representing the excess of deformation without disarrangements over the deformation due to disarrangements. Accordingly, we replace the original energy response $\Psi$ in terms of $G$ and $M$ by the response $\hat{\Psi}$ in terms of the macroscopic deformation gradient $\nabla g$ and the disparity $\Delta$ :

$$
\hat{\Psi}(\nabla g, \Delta):=\Psi\left(\frac{\nabla g+\Delta}{2}, \frac{\nabla g-\Delta}{2}\right) .
$$

The motivation for this choice of variables lies in the version of the stress relation (10) obtained for the new variables :

$$
S=2 D_{F} \hat{\Psi}(F, \Delta) .
$$


Here, as in (9) we use the standard notation $F$ for the macroscopic deformation gradient $\nabla g$, and we denote by $D_{F} \hat{\Psi}$ the partial derivative of $\hat{\Psi}$ with respect to $F$ holding the disparity $\Delta$ fixed.

\section{Submacroscopic stability}

\subsection{Equilibrium structured configurations}

The field equations (5)-(7), and the inequality (8) take the following forms in terms of the macroscopic deformation $g$, the disparity $\Delta=G-M$, and the corresponding free energy response function $\hat{\Psi}$ defined in (11):

$$
\begin{gathered}
\operatorname{div}\left(2 D_{F} \hat{\Psi}\right)+b_{r e f}=0 \\
\left(3 D_{F} \hat{\Psi}-D_{\Delta} \hat{\Psi}\right)(\nabla g)^{T}-\left(D_{F} \hat{\Psi}+D_{\Delta} \hat{\Psi}\right) \Delta^{T}=0 \\
s k\left[\left(D_{F} \hat{\Psi}\right)(\nabla g)^{T}-\left(D_{\Delta} \hat{\Psi}\right) \Delta^{T}\right]=0 \\
\operatorname{det}(\nabla g+\Delta) \leq 8 \operatorname{det} \nabla g .
\end{gathered}
$$

Here, the partial derivatives $D_{F} \hat{\Psi}$ and $D_{\Delta} \hat{\Psi}$ are evaluated at the pair $(\nabla g, \Delta)$. We note also that the stress relation (10) takes the simpler form (12) when the free energy is taken to be a function of $\nabla g$ and $\Delta$.

For a bounded region $B$ with Lipschitz boundary, we write the boundary $\partial B$ as the union of two non-overlapping surfaces $\partial_{p} B$ and $\partial_{t} B$, and we suppose given a macroscopic deformation field $g_{p}$ on $\partial_{p} B$ and traction field $s_{t}$ on $\partial_{t} B$, with $g_{p}$ an injective, point-valued mapping. An equilibrium (structured) configuration for $B$ in the environment $b_{r e f}, g_{p}, s_{t}$ is a structured deformation $(g, \Delta)$ that satisfies the relations (13)-(16) as well as the (mixed) boundary conditions

$$
\begin{gathered}
g(X)=g_{p}(X) \quad \text { for all } X \in \partial_{p} B \\
2 D_{F} \hat{\Psi}(\nabla g(X), \Delta(X)) N(X)=s_{t}(X) \quad \text { for all } X \in \partial_{t} B .
\end{gathered}
$$

Here, $N(X)$ denotes the outward normal to the boundary at the point $X \in \partial_{t} B$. The constitutive relations of the given body imply the stress relation (12), so that the boundary condition (18) expresses the requirement that the tractions on $\partial_{t} B$ associated with the equilibrium configuration $(g, \Delta)$ through (12) coincide with the specified traction field $s_{t}$ on $\partial_{t} B$.

The principal features of this definition lie on the one hand in the formal similarity of the present equilibrium equation (13) and the corresponding equilibrium equation

$$
\operatorname{div} D \Psi+b_{r e f}=0
$$

in the elastostatics of hyperelastic materials: in both equations the stress appears as a derivative of a scalar response function with respect to deformation gradient. On the other hand, an equilibrium configuration in the present setting is subject to satisfaction of the consistency relation (14), the frame-indifference 
condition (15), and the inequality (16) that restrict the macroscopic deformation gradient $F$ and the disparity $\Delta$; in the statics of hyperelastic bodies, these conditions are satisfied identically (Deseri and Owen, 2003) and place no restrictions on the macroscopic deformation.

We note for later use the following relations that are immediate consequences of the additive decomposition (2) and the definitions of the disparity $\Delta$ and the field $K$ :

$$
\begin{gathered}
G=\frac{1}{2}(F+\Delta), \quad M=\frac{1}{2}(F-\Delta), \\
\Delta=2 G-F=F(2 K-I) .
\end{gathered}
$$

\subsection{The augmented energy}

Let an environment $g_{p}, s_{t}$, and $b_{r e f}$ as above, and a point $o$ in space be given. We define the augmented energy of the body in the structured deformation $(g, \Delta)$ by the formula

$$
\mathcal{I}(g, \Delta):=\int_{B}\left(2 \hat{\Psi}(\nabla g, \Delta)-b_{r e f} \cdot g^{o}\right)-\int_{\partial_{t} B} s_{t} \cdot g^{o}
$$

where for each $X \in B, g^{o}(X):=g(X)-o$ is the position vector of the point $g(X)$. (Usually, one writes in place of $g^{o}(X)$ the displacement $u(X):=g(X)-$ $X$; this has the effect of adding a number to the energy that depends only upon the environment and not upon the deformation $(g, \Delta)$.) The number $\mathcal{I}(g, \Delta)$ equals the sum of the "enthalpy" (cf., Truesdell and Noll, 1992, Section 88) $\int_{B}\left(\hat{\Psi}(\nabla g, \Delta)-b_{r e f} \cdot g^{o}\right)-\int_{\partial B_{t}} s_{t} \cdot g^{o}$ and the total Helmholtz free energy $\int_{B} \hat{\Psi}(\nabla g, \Delta)$ of the body in the given environment. The reason that we include the contribution of the Helmholtz free energy twice in defining $\mathcal{I}(g, \Delta)$ is the presence of the factor of two on the right-hand side of the stress relation (12).

We now consider an equilibrium structured configuration $(g, \Delta)$ of $B$ in the given environment as well as a smooth vector field $w$ on $B$ that vanishes on $\partial_{p} B$. The variation of the augmented energy $\mathcal{I}$ with respect to macroscopic deformation for the vector field $w$ is, by definition, the number $\left.\frac{d}{d \eta} \mathcal{I}(g+\eta w, \Delta)\right|_{\eta=0}$. We shall calculate this number under the following provisional assumptions on the referential fields $b_{r e f}$ and $s_{t}$ : at each point $X$ in $B$ the dependence of $b_{r e f}(X)$ on $g(X)$ is smooth and its derivative $D_{g} b_{r e f}$ satisfies

$$
\left(D_{g} b_{r e f}\right)^{T} g^{o}=0
$$

moreover, at each point $X$ in $\partial_{t} B$ the dependence of $s_{t}(X)$ on $g(X)$ is smooth and its derivative $D_{g} s_{t}$ satisfies

$$
\left(D_{g} s_{t}\right)^{T} g^{o}=0
$$

The conditions (22) and (23) admit, among others, the body force field obtained from the standard approximation of the earth's gravitational field at its surface and the surface traction field corresponding to null tractions on $\partial_{t} B$ or, 
more generally, tractions associated with dead loads. Relations (22) and (23) permit us to calculate the variation of the augmented energy with respect to macroscopic deformation as follows:

$$
\left.\frac{d}{d \eta} \mathcal{I}(g+\eta w, \Delta)\right|_{\eta=0}=\int_{B}\left(2 D_{F} \hat{\Psi}(\nabla g, \Delta) \cdot \nabla w-b_{r e f} \cdot w\right)-\int_{\partial_{t} B} s_{t} \cdot w
$$

We add and subtract $\operatorname{div}\left(2 D_{F} \hat{\Psi}(\nabla g, \Delta)\right) \cdot w$ to the volume integral and use the product rule

$$
\operatorname{div}\left(S^{T} w\right)=\operatorname{div} S \cdot w+S \cdot \nabla w
$$

the Divergence Theorem, and the fact that $w$ vanishes on $\partial_{p} B$ to rewrite the variation as

$$
\begin{aligned}
\frac{d}{d \eta} \mathcal{I}(g+\eta w, \Delta) \quad \mid & \eta=0=-\int_{B}\left(\operatorname{div}\left(2 D_{F} \hat{\Psi}(\nabla g, \Delta)\right)+b_{r e f}\right) \cdot w \\
& +\int_{\partial_{t} B}\left(2 D_{F} \hat{\Psi}(\nabla g, \Delta) N-s_{t}\right) \cdot w
\end{aligned}
$$

Because $(g, \triangle)$ is an equilibrium structured configuration, we conclude from (26), (13) and (18) that, for a given environment $g_{p}, s_{t}$, and $b_{r e f}$ and disparity field $\Delta$, the variation $\left.\frac{d}{d \eta} \mathcal{I}(g+\eta w, \Delta)\right|_{\eta=0}$ is zero for every smooth vector field $w$ vanishing on $\partial_{p} B$. Conversely, it is easy to show that if $(g, \Delta)$ is a structured deformation for which $g$ satisfies (17) and if the variation in (26) is zero for every smooth $w$ that vanishes on $\partial_{p} B$, then $(g, \Delta)$ is an equilibrium structured configuration for $B$ in the given environment.

We emphasize that the disparity $\Delta$ is not to be varied when computing the variation of the energy in (26). If one allowed also for variations in $\Delta$, then, in general, the augmented energy would no longer have a stationary value at an equilibrium structured configuration $(g, \Delta)$. It also is important to note that an equilibrium configuration $(g, \Delta)$ not only renders zero the variation of the augmented energy at $(g, \Delta)$ but also satisfies the relations (14)-(16). However, the perturbed configurations $(g+\eta w, \Delta)$ employed in calculating the variation of the augmented energy need not satisfy (14)-(16).

We may write the defining formula (21) in the convenient, abbreviated form

$$
\mathcal{I}=\int_{B}\left(2 \psi-b \cdot g^{o}\right)-\int_{\partial_{t} B} s_{t} \cdot g^{o}
$$

and note from this expression that the augmented energy $\mathcal{I}$ depends upon the given environment and equilibrium structured configuration. The equilibrium structured configuration in turn is determined by the macroscopic deformation field $g$ and any one of the following fields: the disparity $\Delta=G-M$, the deformation without disarrangements $G$, the deformation due to disarrangements $M$, or the field $K=(\nabla g)^{-1} G$ that determines the purely submacroscopic deformation $(i, K)$ from the virgin configuration to the reference configuration. The variety of choices for the companion field to $g$ provides considerable flexibility in the study of "submacroscopically stable equilibria" that follows. 


\subsection{Submacroscopically stable equilibria}

We wish to investigate the possibility that an equilibrium structured configuration of a body can be stable with respect to purely submacroscopic geometrical changes. To this end, we suppose now that at each time $\tau$ in an interval $I$ there is specified an equilibrium structured configuration $\left(g_{\tau}, \Delta_{\tau}\right)$ for the body in a given, time-independent environment $\left(b_{r e f}, s_{t}, g_{p}\right)$, so that the relations (13)(16) and the boundary conditions (17), (18) are satisfied for every time $\tau$. We then call the function $\tau \mapsto\left(g_{\tau}, \Delta_{\tau}\right)$ a quasi-static (structured) process for the body in the time-independent environment $\left(b_{r e f}, s_{t}, g_{p}\right)$. The formula $(27)$ then determines the augmented energy as a function of time $\tau \mapsto \mathcal{I}(\tau)$ and, upon differentiation, yields the formulae for its time derivative $\tau \mapsto \mathcal{I}^{\prime}(\tau)$ :

$$
\begin{aligned}
\mathcal{I}^{\prime} & =\int_{B}\left(2 \psi^{\prime}-b \cdot g^{\prime}\right)-\int_{\partial B_{t}} s_{t} \cdot g^{\prime} \\
& =\int_{B}\left(2 \psi^{\prime}-b \cdot g^{\prime}\right)-\int_{\partial_{t} B} s_{t} \cdot g^{\prime}-\int_{\partial B_{p}} S N \cdot g^{\prime}+\int_{\partial B_{p}} S N \cdot g^{\prime} \\
& =\int_{B}\left(2 \psi^{\prime}-b \cdot g^{\prime}\right)-\int_{\partial_{t} B} S N \cdot g^{\prime}-\int_{\partial B_{p}} S N \cdot g^{\prime}+\int_{\partial B_{p}} S N \cdot\left(g_{p}^{o}\right)^{\prime} \\
& =\int_{B} 2 \psi^{\prime}-\int_{B} b \cdot g^{\prime}-\int_{\partial B} S N \cdot g^{\prime},
\end{aligned}
$$

where we have used the boundary conditions $(17)$, (18), the fact that the environment is time-independent, and the notation ' for $\frac{d}{d \tau}$ in the integrands in the expressions above. For conciseness, we have omitted the subscript $\tau$ on the time-dependent fields in the last formula. The equilibrium equation (13) and the Divergence Theorem yield immediately the formula

$$
\mathcal{I}^{\prime}=\int_{B}\left(2 \psi^{\prime}-S \cdot \nabla g^{\prime}\right)
$$

If we assume further that the dissipation inequality

$$
\psi^{\prime} \leq S \cdot \nabla g^{\prime}
$$

holds throughout the body at every time, then the dissipation rate $S \cdot \nabla g^{\prime}-\psi^{\prime}$ is non-negative and (28) becomes

$$
\mathcal{I}^{\prime}=\int_{B} \psi^{\prime}-\int_{B}\left(S \cdot \nabla g^{\prime}-\psi^{\prime}\right) .
$$

We conclude that in quasi-static processes proceeding in a time-independent environment and satisfying the dissipation inequality, the rate of change of augmented energy equals the rate of storage of energy $\int_{B} \psi^{\prime}$ minus the rate of dissipation of energy $\int_{B}\left(S \cdot \nabla g^{\prime}-\psi^{\prime}\right)$. Moreover, the dissipation inequality (29) together with the calculations that produced (28) yield the energy inequalities

$$
\mathcal{I}^{\prime} \leq \int_{B} \psi^{\prime} \leq \int_{B} S \cdot \nabla g^{\prime}=\int_{B} b \cdot g^{\prime}+\int_{\partial B} S N \cdot g^{\prime}
$$


i.e., under the above assumptions, the rate of change of augmented energy does not exceed the rate of change of the free energy which, in turn, does not exceed the power expended by the (time-independent) environment.

Let us apply these considerations in the special case in which the geometrical changes in the body occur only at submacroscopic levels, so that the macroscopic deformation $g_{\tau}$ is independent of $\tau$, i.e., $g^{\prime}=0$. The rate of dissipation of energy then is $-\int_{B} \psi^{\prime}$, which equals minus the rate of storage of energy, and (30) becomes $\mathcal{I}^{\prime}=2 \int_{B} \psi^{\prime} \leq 0$. Thus, in purely submacroscopic, quasistatic processes in a time-independent environment, the dissipation inequality implies that

1. the augmented energy cannot increase, and, by (30),

2. the body dissipates energy at the rate $-\int_{B} \psi^{\prime}$, the same rate at which its free energy decreases.

Item 1 prompts us, following the methodology of Gibbs, to single out for consideration those structured equilibrium configurations $(g, \Delta)$ such that purely submacroscopic processes that start at $(g, \Delta)$ cannot decrease the augmented energy. Accordingly, we shall seek equilibrium configurations $(g, \Delta)$ satisfying $\mathcal{I}(g, \Delta) \leq \mathcal{I}(g, \tilde{\Delta})$ for all tensor fields $\tilde{\Delta}$ in a suitable collection $\mathcal{D}_{g}$.

There is some freedom in the choice of the collection $\mathcal{D}_{g}$, and it is reasonable to require at least that the pairs $(g, \tilde{\Delta})$ with $\tilde{\Delta}$ in $\mathcal{D}_{g}$ are structured deformations and, hence, satisfy the inequality (16). In order that such structured deformations $(g, \tilde{\Delta})$ can occur in the given body, it is necessary that they also satisfy the consistency relation (14) and the frame-indifference condition (15). In other words, the imposition of (14) and (15) rule out competitors $(g, \tilde{\Delta})$ that only arise as deformations of a body composed of a different material. It remains to decide whether or not to require that these competing structured deformations also satisfy the equilibrium equation (13) and the traction boundary condition (18). We choose here to make the collection $\mathcal{D}_{g}$ as large as possible, and, accordingly, we impose neither the equilibrium equation (13) nor the traction boundary condition (18). This choice risks passing over interesting competitors that would minimize the augmented energy over a proper subcollection of $\mathcal{D}_{g}$ but not over the entire collection $\mathcal{D}_{g}$. This risk is worthwhile if the collection provides a rich variety of minimizers, and the final results should be examined in this light.

These considerations lead us, for each macroscopic deformation $g$ of $B$ that satisfies the placement boundary condition (17), to define the collection

$$
\mathcal{D}_{g}:=\{\tilde{\Delta}: B \rightarrow \operatorname{Lin} \mid(g, \tilde{\Delta}) \text { satisfies (14), (15), (16) }\} .
$$

We may now define the central concept in this study: an equilibrium structured configuration $(g, \Delta)$ for $B$ in a given environment is said to be submacroscopically stable if

$$
\mathcal{I}(g, \Delta) \leq \mathcal{I}(g, \tilde{\Delta}) \text { for all tensor fields } \tilde{\Delta} \text { in } \mathcal{D}_{g},
$$


with $\mathcal{I}(g, \Delta)$ defined in (21). This terminology is motivated by the above consequence of the energy inequality (31): quasistatic, purely submacroscopic processes in a time-independent environment cannot increase the augmented energy of the body. This result suggests that submacroscopically stable equilibria may be approached or attained in purely submacroscopic quasistatic processes and may be exited only exceptionally during such processes, namely, when the augmented energy remains at its minimum value as the body changes submacroscopically.

A different notion of submacroscopic stability was introduced by Deseri and Owen, 2003, that reflects only the behavior of the Helmholtz free energy density near a given pair of tensors $(F, \Delta)$ or, more precisely, near $(F, G)$, and that does not impose the conditions (13) - (15) on submacroscopic competitors.

When a structured deformation is described by means of the macroscopic deformation $g$ and one of the tensor fields $G=\frac{1}{2}(\nabla g+\Delta), M=\frac{1}{2}(\nabla g-\Delta)$, or (c.f. $(20))$

$$
K=(\nabla g)^{-1} G=\frac{1}{2}\left(I+(\nabla g)^{-1} \Delta\right),
$$

then the augmented energy may be expressed as a function of $g$ and the chosen tensor field, and satisfaction of (33) is then equivalent to satisfaction of a corresponding inequality in terms of $g$ and the chosen tensor field. For example, if $K$ is the chosen field, then the inequality (33) is equivalent to

$$
\overline{\mathcal{I}}(g, K) \leq \overline{\mathcal{I}}(g, \tilde{K}) \text { for all tensor fields } \tilde{K} \text { in } \overline{\mathcal{D}_{g}},
$$

where now $\overline{\mathcal{D}_{g}}$ is the set of continuous tensor fields $K$ satisfying the consistency relation (14) in the equivalent form

$$
D_{F} \bar{\Psi}\left(2 K^{-T}-I\right)+(\nabla g)^{-T} D_{K} \bar{\Psi}\left\{K^{-T}-3 I+K^{T}\right\}=0,
$$

satisfying the frame-indifference relation (15) in the equivalent form

$$
\operatorname{sk}\left(D_{F} \bar{\Psi} F^{T}+F^{-T} D_{K} \bar{\Psi}\left(I-2 K^{T}\right) F^{T}\right)=0,
$$

and satisfying the determinant inequality (16) in the equivalent form

$$
0<\operatorname{det} K \leq 1
$$

(See [?], relations (10.24), (10.25), and (10.27).) In the relations (35) and (36), $\bar{\Psi}$ denotes the free energy expressed as a function of the variables $F=\nabla g$ and $K$, and $D_{F} \bar{\Psi}$ and $D_{K} \bar{\Psi}$ denote its partial derivatives. This notation permits us to recast the definition (21) in the equivalent form

$$
\overline{\mathcal{I}}(g, K):=\int_{B}\left(2 \bar{\Psi}(\nabla g, K)-b_{r e f} \cdot g^{o}\right)-\int_{\partial_{t} B} s_{t} \cdot g^{o}
$$

We mention in closing this section that the conclusions about the augmented energy and its rate of change, stated in italics in the preceding text, do not rest on the satisfaction of the consistency relation (14), the frame-indifference relations (15), and the inequality (16). Moreover, those conclusions do not require the provisional assumptions (22) and (23) made in calculating the variation of the augmented energy with respect to macroscopic deformation. 


\subsection{A procedure for determining submacroscopically sta- ble equilibria}

The discussion above suggests how one can search for submacroscopically stable equilibria. As a first step, one may ignore the requirement that a submacroscopically stable equilibrium configuration satisfy the balance equation (13) and the traction boundary condition (18). Accordingly, one may fix a macroscopic deformation $g$ that satisfies the boundary condition (17) on $\partial_{p} B$ and make a particular choice among the tensor variables $\Delta, G, M$, and $K$ that facilitates the solutions of the consistency relation (14), the frame-indifference relation (15), and the inequality (16). We assume for the sake of definiteness that the variable $K$ has been chosen, so that (35), (36), and (37) are used in place of (14), (15), and (16). One then seeks to determine at least one energetically preferred phase $K_{s s}$ for the body in the given macroscopic configuration $g$, i.e., one looks for a tensor field $K=K_{s s}$ satisfying the inequality (34) in which the augmented energy is given by the formula (38). Such a field $K_{s s}$ would represent an energetically preferred microgeometry in the reference configuration for the body, a geometry attained via the purely submacroscopic deformation $\left(i, K_{s s}\right)$ starting in the virgin configuration of the body.

The formula (38) for the augmented energy in terms of the fields $g$ and $K$ shows that minimization of the augmented energy for the given macroscopic deformation $g$ and boundary traction field $s_{t}$ amounts to minimizing pointwise the integrand $\bar{\Psi}(\nabla g, K)$ on the right-hand side of (38) with respect to the tensor variable $K$, subject to the satisfaction of (35), (36), and (37). Consequently, the first step in determining partially relaxed equilibrium configurations reduces to the minimization, for a fixed tensor $F=\nabla g$, of the free energy density $K \mapsto \bar{\Psi}(F, K)$ subject to $(35),(36)$, and (37). We refer to this as the first-step minimization problem.

In cases where solving the first step minimization problem provides a definite function $F \mapsto K_{s s}(F)$, the original stress relation (12) then provides a relation in which the stress $S$ is a function $F \mapsto S_{s s}(F)$ of the deformation gradient $F=\nabla g$; similarly, the original constitutive relation for the volume density $\psi$ of Helmholtz free energy in the reference configuration, as a function $F \mapsto \Psi_{s s}(F)$ of $F$ and $K$, provides a relation in which $\psi$ is a function of $F$. In this case, the first step minimization problem provides a Cauchy elastic body whose response functions for stress $S_{s s}$ and Helmholtz free energy density $\Psi_{s s}$ are determined by the original response function $\bar{\Psi}$ alone. We note that, in every deformation process of this Cauchy elastic body, the dissipation inequality must hold, because each such process corresponds to a process of the original body undergoing disarrangements, and the dissipation inequality is satisfied in all processes of that body.

In the second step of the search for submacroscopically stable equilibria, one seeks equilibrium configurations of the form $\left(g, K_{s s}\right)$, and one imposes, in addition to the placement boundary condition (17) on $g$, the equilibrium equation (13) as well as the boundary condition (18) in order to determine the macroscopic deformation $g$, itself. In cases where $K_{s s}$ is a well-defined function 
of $\nabla g$, this amounts to finding an equilibrium configuration $g$ for the non-linearly Cauchy elastic body with stress response $F \mapsto S_{s s}(F)$.

In the following two sections we carry out the first step in detail for two specific choices of free energy response function, and we briefly discuss for the second step the resulting boundary value problem for $g$.

\section{Example: biquadratic energy $\Psi_{K O}$}

A fruitful setting in which to study partially relaxed configurations is a body having the bi-quadratic free energy function

$$
\Psi_{K O}(G, M)=\frac{1}{2} \alpha_{\backslash}|G|^{2}+\frac{1}{2} \alpha_{d}|M|^{2},
$$

studied by Kirby and Owen, 2005. Here, we take the material constant $\alpha_{\backslash}$ to be positive and allow the remaining material constant $\alpha_{d}$ to be any non-zero real number. Because

$$
D_{M} \Psi_{K O}(G, M) M^{T}=\alpha_{d} M M^{T}
$$

is symmetric, the consistency relation (6) implies that (7) automatically is satisfied, so that the frame-indifference condition (7) need not be considered further. Consequently, a structured deformation $(g, G)$ determines an equilibrium configuration if and only if it satisfies (5), (6), (8), and (9). The formula (39) tells us that the equilibrium equation (5) and consistency relation (6) now take the forms

$$
\begin{gathered}
\operatorname{div}\left(\alpha_{\backslash} G+\alpha_{d} M\right)+b_{r e f}=0 \\
\alpha_{\backslash} G M^{T}+\alpha_{d} M(G+M)^{T}=0,
\end{gathered}
$$

and the stress relation (10) becomes

$$
S=\alpha_{\backslash} G+\alpha_{d} M .
$$

\subsection{Portfolio of phases}

\subsubsection{Solutions of the consistency relations}

The additive decomposition (9) and the definition $K=F^{-1} G$ permit us to rewrite the consistency relation (41) in terms of the fields $F=\nabla g$ and $K$ :

$$
\begin{aligned}
0 & =\alpha_{\backslash}(F K)(F-F K)^{T}+\alpha_{d}(F-F K) F^{T} \\
& =\alpha_{\backslash} F\left(K-K K^{T}\right) F^{T}+\alpha_{d} F(I-K) F^{T} \\
& =F\left[\alpha_{\backslash}\left(K-K K^{T}\right)+\alpha_{d}(I-K)\right] F^{T} \\
& =F\left[\left(\alpha_{\backslash}-\alpha_{d}\right) K-\alpha_{\backslash} K K^{T}+\alpha_{d} I\right] F^{T} .
\end{aligned}
$$

The values of the field $F$ are invertible, so that the consistency relation is equivalent to the following equation that involves the field $K$ alone:

$$
\left(\alpha_{\backslash}-\alpha_{d}\right) K-\alpha \backslash K K^{T}+\alpha_{d} I=0 .
$$


We shall now follow [?] and obtain all the solutions $K$ of the consistency relation (43) that satisfy the determinant inequality (1), now expressed in terms of $K$ :

$$
0<\operatorname{det} K \leq 1
$$

We consider first the case $\alpha_{\backslash}=\alpha_{d}$. Because both material constants are non-zero, the consistency relation reduces to $K K^{T}=I$, and the determinant inequality implies that the values $K(X)$ of the field $K$ in this case are arbitrary proper orthogonal tensors:

$$
K(X) \in O r t h^{+} \ldots \text { textured phase at } X \text {. }
$$

For the case $\alpha_{\backslash} \neq \alpha_{d}$, the consistency relation tells us that the values of the field $K$ are symmetric, i.e., $K=K^{T}$, and (43) is then equivalent to

$$
\left(K+\frac{1}{\nu} I\right)(K-I)=K^{2}-\left(1-\frac{1}{\nu}\right) K-\frac{1}{\nu} I=0,
$$

where

$$
\nu:=\frac{\alpha_{\backslash}}{\alpha_{d}} \in(-\infty, 0) \cup(0, \infty) .
$$

The symmetry of $K$ and (46) tell us that at each point $X$ of the body the spectrum of $K(X)$ contains only the numbers 1 and $-\frac{1}{\nu}$, and the determinant inequality (44) implies that there are exactly four possibilities for the spectrum of $K(X):(1,1,1)$ with $\nu$ non-zero, $\left(-\frac{1}{\nu}, 1,1\right)$ with $\nu \leq-1,\left(-\frac{1}{\nu},-\frac{1}{\nu}, 1\right)$ with $|\nu| \geq 1$, and $\left(-\frac{1}{\nu},-\frac{1}{\nu},-\frac{1}{\nu}\right)$ with $\nu \leq-1$. The corresponding spectral representations of $K(X)$ are:

$$
\begin{gathered}
K(X)=I \ldots \text { classical phase at } X \quad(\nu \neq 0), \\
K(X)=I-\frac{\nu+1}{\nu} e(X) \otimes e(X) \ldots \text { oblate phase at } X \quad(\nu \leq-1), \\
K(X)=-\frac{1}{\nu} I+\frac{\nu+1}{\nu} e(X) \otimes e(X) \ldots \text { prolate phase at } X \quad(|\nu| \geq 1), \\
K(X)=-\frac{1}{\nu} I \ldots \text { spherical phase at } X \quad(\nu \leq-1) .
\end{gathered}
$$

Because $F$ and $G$ are continuous fields, it follows that $K=F^{-1} G$ is continuous. The requirements that $K$ be a continuous field and satisfy the consistency relation (43) imply for $|\nu| \neq 1$ that $K(X)$ must satisfy one and the same relation among (45), (48) - (51) as $X$ varies throughout $B$. For the case $\nu=1$, the classical phase is a special case of the textured phase, and relations (45) and (50) imply that $K(X)$ must satisfy one and the same relation, (45) or (50), at every $X$ in $B$. Finally, when $\nu=-1$, the four phases (48) - (51) are the same and $K(X)=I$ throughout $B$. Thus, continuity of $K$ and satisfaction of consistency in the case of $\Psi_{K O}$ imply that every point of the body is in the same phase, so that our present treatment does not cover phase mixtures. We indicate in Section 6 circumstances that would entail the consideration of mixtures of phases. 
These comments lead to the following terminology: the body is in the classical phase if $K=I$; the body is in the oblate phase if $\nu \leq-1$ and there exists a continuous unit vector field $e$ on the closure of $B$ such that $K$ is given by (49) for all $X \in B$. We say the body is in the prolate phase if $|\nu| \geq 1$ and there exists a continuous unit vector field $e$ on the closure of $B$ such that $K$ is given by (50) for all $X \in B$. The body is in the spherical phase if $\nu \leq-1$ and $K(X)=-\frac{1}{\nu} I$ for all $X \in B$.

\subsubsection{Submacroscopic geometry of the phases}

In the oblate phase when $\nu<-1, e(X)$ is a unit eigenvector of $K(X)$ corresponding to the eigenvalue $-\frac{1}{\nu} \in(0,1)$ while directions perpendicular to $e(X)$ are eigenvectors corresponding to the eigenvalue 1 . Therefore, the deformation without disarrangements corresponding to the purely submacroscopic deformation $(i, K)$ causes shortening to occur in the direction $e(X)$, while no change occurs to vectors orthogonal to $e(X)$. In other words, a small cube centered at $X$ with a face normal to $e(X)$ is flattened in that direction, is unchanged in orthogonal directions, and takes the shape of a thin plate (uniaxial shortening). When $\nu=-1$, the oblate phase reduces to the classical phase.

For the prolate phase, $e(X)$ is a unit eigenvector of $K(X)$ corresponding to the eigenvalue 1 , while vectors in directions perpendicular to $e(X)$ are eigenvectors corresponding to the eigenvalue $-\frac{1}{\nu}$. The deformation without disarrangements corresponding to the purely submacroscopic deformation $(i, K)$ causes no change to occur in the direction $e(X)$, whereas, for $\nu<-1$, shortening alone occurs in directions perpendicular to $e(X)$. When $\nu>1$, shortening again occurs in directions perpendicular to $e(X)$, but now followed by a rotation of amount $\pi$ about $e(X)$. Geometrically, a small cube centered at $X$ with a face normal to $e(X)$ is unchanged in that direction, but is flattened in the directions of the orthogonally oriented faces, and takes the shape of a thin rod (biaxial shortening). When $\nu=-1$, the prolate phase reduces to the classical one, while, when $\nu=1$, the prolate phase reduces to the textured phase.

For the spherical phase, every non-zero vector is an eigenvector of $K(X)$ corresponding to eigenvalue $-\frac{1}{\nu}$. When $\nu<-1$, the deformations without disarrangement for $(i, K)$ cause shortening to occur in every direction, so that a small cube is transformed into a smaller cube (triaxial shortening); when $\nu=-1$, the spherical phase reduces to the classical one.

The consistency relation (43) and the determinant inequality (44) thus determine five phases, the phases for the elastic body with energy $\Psi_{K O}$ or, more briefly, the $\Psi_{K O}$-phases: textured, classical, oblate, prolate, and spherical . For each phase, the structured deformation $(i, K)$ is the purely submacroscopic deformation that takes the body from the virgin configuration to the reference configuration; the classical deformation $(g, \nabla g)$ then takes the body to the current (or deformed) configuration. The Approximation Lemma (Del Piero and Owen, 1993) shows that there is a sequence $n \mapsto h_{n}$ of injective, piecewise affine mappings such that $\lim _{n \rightarrow \infty} h_{n}=i$ and $\lim _{n \rightarrow \infty} \nabla h_{n}=K$. For example, in the case of the spherical phase, for a given positive integer $n$, the mapping $h_{n}$ can 
be taken as one that divides the body into cubic cells of edge proportional to $n^{-1}$ and that performs a triaxial shortening on each cell individually, holding the center of each cell fixed, and thereby reducing the volume of each cell by the factor $-\nu^{-3} \in(0,1)$. The mapping $h_{n}$ also creates voids due to the shortening of adjacent cubic cells. In this example, the deformation without disarrangements for the purely submacroscopic structured deformation $(i, K)$ is the field $K=\lim _{n \rightarrow \infty} \nabla h_{n}=-\frac{1}{\nu} I$, itself. The deformation due to disarrangements is the field $\nabla i-K=I-K=\frac{\nu+1}{n u} I$ that reflects, in the limit as $n$ tends to infinity, the discontinuities of each field $h_{n}$ across the interfaces of adjacent cubic cells. The disarrangements described by the field $\nabla i-K=\frac{\nu+1}{r} I$ reflect the creation of voids that accompanies the triaxial shortening of each cubic cell under the action of $h_{n}$.

It is important to keep in mind that in the textured, oblate, and prolate phases, $K(X)$ can vary with $X$, due to the variation of the orthogonal tensor $K(X)$ for the textured phase or due to the variation of the unit vector $e(X)$ for the prolate and oblate phases; in the classical and spherical phases, $K(X)$ does not vary with $X$.

\subsubsection{The augmented energy in each $\Psi_{K O}$-phase}

In order to obtain explicit formulas for the augmented energy in each $\Psi_{K O^{-}}$ phase, it is convenient to rewrite the formula (21) for the augmented energy in the following form

$$
\overline{\mathcal{I}}_{K O}(g, K)=\int_{B}\left(2 \bar{\Psi}_{K O}(\nabla g, K)-b_{r e f} \cdot g^{o}\right)-\int_{\partial_{t} B} s_{t} \cdot g^{o}
$$

where $\bar{\Psi}_{K O}(\nabla g, K)$ denotes the Helmholtz free energy, expressed as a function of $F=\nabla g$ and $K$. The relations $G=K F$ and $M=F(I-K)$ when substituted into (39) yield the formulas

$$
\begin{aligned}
\bar{\Psi}_{K O}(F, K) & =\frac{\alpha_{\backslash}}{2} F K \cdot F K+\frac{\alpha_{d}}{2} F(I-K) \cdot F(I-K) \\
& =\frac{1}{2} F^{T} F \cdot\left(\left(\alpha_{\backslash}+\alpha_{d}\right) K K^{T}+\alpha_{d}\left(I-K-K^{T}\right)\right) .
\end{aligned}
$$

For each of the two cases $\alpha_{\backslash}=\alpha_{d}$ and $\alpha_{\backslash} \neq \alpha_{d}$, the consistency relation (43) then implies

$$
\bar{\Psi}_{K O}(F, K)=\frac{\alpha \backslash}{2 \nu^{2}} F^{T} F \cdot\left(\left(\nu^{2}-2 \nu-1\right) K+(2 \nu+1) I\right) .
$$

When the relations $G=K F$ and $M=F(I-K)$ are substituted into the stress relation (42), that relation becomes

$$
\begin{aligned}
\bar{S}_{K O}(\nabla g, K) & =\alpha \backslash F K+\alpha_{d} F(I-K) \\
& =\frac{\alpha \backslash}{\nu} F((\nu-1) K+I) .
\end{aligned}
$$


The relations (52) and (53) then imply

$$
\begin{aligned}
& \overline{\mathcal{I}}_{K O}(g, K)+\int_{B}\left(b_{r e f} \cdot g^{o}-\alpha \backslash \frac{2 \nu+1}{\nu^{2}} \operatorname{tr}\left(F^{T} F\right)\right)+\int_{\partial_{t} B} s_{t} \cdot g^{o} \\
& =\int_{B} \frac{\nu^{2}-2 \nu-1}{\nu^{2}} \alpha \backslash F^{T} F \cdot K .
\end{aligned}
$$

This formula shows that, for given boundary data $g_{p}$ on $\partial_{p} B$ and $s_{t}$ on $\partial_{t} B$, for a given body force field $b_{\text {ref }}$ on $B$, and for a given macroscopic deformation field $g$ that agrees with $g_{p}$ on $\partial B_{p}$, the determination of a structured deformation $(g, K)$ satisfying (34) reduces now to the minimization of the integrand $\frac{\nu^{2}-2 \nu-1}{\nu^{2}} \alpha \backslash F^{T} F \cdot \tilde{K}$, with $F=\nabla g$ fixed and with $\tilde{K}$ any one of the $\Psi_{K O^{-}}$ phases of the body determined in the previous subsection. We refer to this minimization problem as the pure-phase minimization of the augmented energy.

Of course, each candidate $(g, K)$ for a submacroscopically stable equilibrium configuration must not only be a solution of the pure-phase minimization problem but also must satisfy balance of forces (13) and the traction boundary condition (18), which we record here in terms of the fields $g$ and $K$ in the case of the free energy response $\Psi_{K O}$ (see Deseri and Owen, 2003, (10.23), (10.28) for the case of a general free energy response $\bar{\Psi}$ ):

$$
\begin{gathered}
\operatorname{div}\left(\frac{\alpha}{\nu} \nabla g((\nu-1) K+I)\right)+b_{r e f}=0 \\
\frac{\alpha_{\backslash}}{\nu} \nabla g((\nu-1) K+I) N=s_{t} \quad \text { on } \partial_{t} B .
\end{gathered}
$$

We shall impose the requirements (56) and (57) after we undertake the purephase minimization of the augmented energy.

\subsection{Pure-phase energy minimization within each $\Psi_{K O}$-phase}

For each $\Psi_{K O}$-phase of the body undergoing the macroscopic deformation $g$, we now determine the structured deformations $(g, K)$ (if any) that accomplish the pure-phase minimization of the augmented energy, and we determine the minimum value (if any) of the augmented energy for each phase.

\subsubsection{Classical, spherical, and textured phases}

According to (48), for the classical phase the identity field $K=I$ is the only competitor, and, by (55), the minimum augmented energy is given by

$$
\overline{\mathcal{I}}_{K O}^{\text {class }}(g):=\overline{\mathcal{I}}_{K O}(g, I)=\int_{B}\left(\alpha \backslash \operatorname{tr} C-b_{\text {ref }} \cdot g^{o}\right)-\int_{\partial B_{t}} s_{t} \cdot g^{o}
$$

where $C:=F^{T} F$; the stress in (54) is given by

$$
\bar{S}_{K O}^{\text {class }}(F):=\bar{S}_{K O}(F, I)=\alpha \backslash F \text {. }
$$


For the spherical phase, the relation (51) tells us that $K=-\frac{1}{\nu} I$ is the only competitor, that the minimum augmented energy is given via (55) by

$$
\begin{aligned}
\overline{\mathcal{I}}_{K O}^{s p h}(g): & =\overline{\mathcal{I}}_{K O}\left(g,-\frac{1}{\nu} I\right) \\
& =\int_{B}\left(\frac{\nu^{2}+3 \nu+1}{\nu^{3}} \alpha \backslash \operatorname{tr} C-b_{r e f} \cdot g^{o}\right)-\int_{\partial_{t} B} s_{t} \cdot g^{o},
\end{aligned}
$$

and that the stress is given by

$$
\bar{S}_{K O}^{s p h}(F):=\bar{S}_{K O}\left(F,-\frac{1}{\nu} I\right)=\frac{1}{\nu^{2}} \alpha \backslash F .
$$

In these formulas, the ratio $\nu$ does not exceed -1 .

In the case of the textured phase, the ratio $\nu$ equals 1 , every proper orthogonalvalued field $K$ is a competitor, and (55) becomes

$$
\overline{\mathcal{I}}_{K O}(F, K)+\int_{B} b_{r e f} \cdot g^{o}+\int_{\partial_{t} B} s_{t} \cdot g^{o}=\int_{B} \alpha \backslash C \cdot(3 I-2 K) .
$$

Writing the positive definite, symmetric tensor field $C=F^{T} F$ in its spectral representation $\sum_{i} c_{i} e_{i} \otimes e_{i}$ with $e_{1}, e_{2}, e_{3}$ orthonogonal unit vector fields, we have

$$
\begin{aligned}
C \cdot(3 I-2 K) & =3 \operatorname{tr} C-2 \operatorname{tr}(C K) \\
& =3 \sum_{i} c_{i}-2 \sum_{i} c_{i} K e_{i} \cdot e_{i} \\
& \geq 3 \sum_{i} c_{i}-2 \sum_{i} c_{i}=\operatorname{tr} C,
\end{aligned}
$$

where we have used the fact that $c_{i}>0$ and, because $K$ is orthogonal-valued, $K e_{i} \cdot e_{i} \leq 1$. It follows that the integrand for the volume integral on the righthand side of (62) is minimized when $K$ is the constant field $I$, so that the minimum augmented energy for the textured phase is given by:

$$
\begin{aligned}
\overline{\mathcal{I}}_{K O}^{\text {text }(g):} & =\overline{\mathcal{I}}_{K O}(g, I)= \\
& =\int_{B}\left(\alpha \backslash \operatorname{tr} C-b_{\text {ref }} \cdot g^{o}\right)-\int_{\partial_{t} B} s_{t} \cdot g^{o}=\overline{\mathcal{I}}_{K O}^{\text {class }}(F) .
\end{aligned}
$$

We note also that the stress in the textured phase (whether or not $K=I$ ) is given by

$$
\bar{S}_{K O}^{\text {text }}(F):=\alpha \backslash F=\bar{S}_{K O}^{\text {class }}(F)
$$

\subsubsection{Prolate and oblate phases}

According to (49), in the oblate phase we have $\tilde{K}=I-\frac{\nu+1}{r} \tilde{e} \otimes \tilde{e}$ (with $\tilde{e}$ a continuous unit vector field on the closure of $B$ ), and the right-hand side of the 
relation (55) is given by

$$
\begin{aligned}
\int_{B} \frac{\nu^{2}-2 \nu-1}{\nu^{2}} \alpha_{\backslash} C \cdot \tilde{K} & \\
& =\int_{B} \frac{\nu^{2}-2 \nu-1}{\nu^{2}} \alpha \backslash \operatorname{tr} C-\int_{B} \frac{P(\nu)}{\nu^{3}} \alpha \backslash C \tilde{e} \cdot \tilde{e}
\end{aligned}
$$

where $P(\nu)=\left(\nu^{2}-2 \nu-1\right)(\nu+1)=\nu^{3}-\nu^{2}-3 \nu-1$. This formula and the relation (55) give another expression for the augmented energy in the oblate phase

$$
\overline{\mathcal{I}}_{K O}\left(g, I-\frac{\nu+1}{\nu} \tilde{e} \otimes \tilde{e}\right)=\int_{B}\left(\alpha \backslash\left[t r C-\frac{P(\nu)}{\nu^{3}} C \tilde{e} \cdot \tilde{e}\right]-b_{r e f} \cdot g^{o}\right)-\int_{\partial B_{t}} s_{t} \cdot g^{o} .
$$

Because $\nu \leq-1$ for the oblate phase, the number $P(\nu)$ is not positive. Therefore, $-P(\nu) / \nu^{3}$ is not positive, and the integrand for the volume integral in (65) is minimized when the unit vector field $\tilde{e}$ maximizes $C \tilde{e} \cdot \tilde{e}$, i.e., when the value of $\tilde{e}$ at each point $X$ is an eigenvector $e_{\max }$ of $C(X)=F(X)^{T} F(X)$ corresponding to the maximum eigenvalue $c_{\max }(X)$ of $C(X)$. Therefore, because $c_{\max }$ is an integrable function on $B$ (Silhavy, 1997), we have

$$
-\int_{B} \frac{P(\nu)}{\nu^{3}} \alpha \backslash C \tilde{e} \cdot \tilde{e} \geq-\int_{B} \frac{P(\nu)}{\nu^{3}} \alpha \backslash c_{\max },
$$

and the minimum value of the augmented energy for the case of the oblate phase is given by

$$
\begin{aligned}
\overline{\mathcal{I}}_{K O}^{o b l}(g): & =\overline{\mathcal{I}}_{K O}\left(g, I-\frac{\nu+1}{\nu} e_{\max } \otimes e_{\max }\right) \\
& =\int_{B}\left(\alpha_{\backslash}\left(t r C-\frac{P(\nu)}{\nu^{3}} c_{\max }\right)-b_{r e f} \cdot g^{o}\right)-\int_{\partial_{t} B} s_{t} \cdot g^{o} .
\end{aligned}
$$

At each point $X$ of $B$, the unit vector $e_{\max }(X)$ in the last formula depends upon the macroscopic deformation gradient $F(X)$ through the condition that $e_{\max }(X)$ is a principal direction of $C(X)$ corresponding to the maximum eigenvalue of $C(X)$, although in some cases the diad $e_{\max }(X) \otimes e_{\max }(X)$ need not be completely determined by $F(X)$. It is useful to record here the expression for the stress $S_{K O}^{o b l}(F, K)$ when $K=I-\frac{\nu+1}{\nu} e_{\max } \otimes e_{\max }$ :

$$
S_{K O}^{o b l}\left(F, I-\frac{\nu+1}{\nu} e_{\max } \otimes e_{\max }\right)=\alpha \backslash F\left(I+\frac{1-\nu^{2}}{\nu^{2}} e_{\max } \otimes e_{\max }\right) .
$$

We note that the stress given in (68) need not be completely determined by the deformation gradient $F$, while the volume integral on the right-hand side of (67) is completely determined by the field $F$.

According to (50), in the prolate phase we have $\tilde{K}=-\frac{1}{\nu} I+\frac{\nu+1}{\nu} \tilde{e} \otimes \tilde{e}$ (with $\tilde{e}$ a continuous unit vector field on the closure of $B$ ), and the right-hand side of 
the relation (55) is given by

$$
\begin{aligned}
\int_{B} \frac{\nu^{2}-2 \nu-1}{\nu^{2}} \alpha_{\backslash} C \cdot \tilde{K} & =\int_{B} \frac{\nu^{2}-2 \nu-1}{\nu^{2}} \alpha \backslash C \cdot\left(-\frac{1}{\nu} I+\frac{\nu+1}{\nu} \tilde{e} \otimes \tilde{e}\right) \\
& =-\int_{B} \frac{\nu^{2}-2 \nu-1}{\nu^{2}} \alpha \backslash \operatorname{tr} C+\int_{B} \frac{P(\nu)}{\nu^{3}} \alpha \backslash C \cdot \tilde{e} \otimes \tilde{e} \\
& =-\int_{B} \frac{\nu^{2}-2 \nu-1}{\nu^{2}} \alpha \backslash \operatorname{tr} C+\int_{B} \frac{P(\nu)}{\nu^{3}} \alpha \backslash C \tilde{e} \cdot \tilde{e}
\end{aligned}
$$

where, as in the case of the oblate phase, $P(\nu)=\left(\nu^{2}-2 \nu-1\right)(\nu+1)=$ $\nu^{3}-\nu^{2}-3 \nu-1$. This formula and the relation (55) give the alternative expression for the augmented energy in the prolate phase

$$
\begin{aligned}
\overline{\mathcal{I}}_{K O}\left(g,-\frac{1}{\nu} I+\frac{\nu+1}{\nu} \tilde{e} \otimes \tilde{e}\right) & =\int_{B} \alpha \backslash\left(\frac{\nu^{2}+3 \nu+1}{\nu^{3}} \operatorname{tr} C+\frac{P(\nu)}{\nu^{3}} C \tilde{e} \cdot \tilde{e}\right) \\
& -\int_{B} b_{r e f} \cdot g^{o}-\int_{\partial_{t} B} s_{t} \cdot g^{o} .
\end{aligned}
$$

The fraction $\frac{\nu^{3}-\nu^{2}-3 \nu-1}{\nu^{3}}=P(\nu) / \nu^{3}$ for $|\nu| \geq 1$ is positive on the intervals $-\infty<\nu<-1$ and $1+\sqrt{2}<\nu$ and is negative on the interval $1<\nu<1+\sqrt{2}$. Consequently, because the eigenvalues $c_{\min }, c_{\text {mid }}$, and $c_{\max }$ of $C$ are integrable on $B$, the formula (69) provides the following formulas for the minimum augmented energy in the prolate phase $\overline{\mathcal{I}}_{K O}^{\text {pro }}(g)$ :

$$
\begin{aligned}
& \overline{\mathcal{I}}_{K O}^{\text {pro }}(g)+\int_{B} b_{\text {ref }} \cdot g^{o}+\int_{\partial_{t} B} s_{t} \cdot g^{o} \\
= & \left\{\begin{array}{cc}
\int_{B} \frac{\alpha_{\backslash}}{\nu^{3}}\left\{\nu^{3} c_{\text {min }}+\left(\nu^{2}+3 \nu+1\right)\left(c_{\text {mid }}+c_{\max }\right)\right\} & \text { if } \nu \notin[-1,1+\sqrt{2}] \\
\int_{B} \frac{\alpha_{\backslash}}{\nu^{3}}\left\{\left(\nu^{2}+3 \nu+1\right)\left(c_{\text {min }}+c_{\text {mid }}\right)+\nu^{3} c_{\max }\right\} & \text { if } 1<\nu<1+\sqrt{2}) .
\end{array}\right.
\end{aligned}
$$

The integrand on the right-hand side of this relation provides an expression that for $1<\nu<1+\sqrt{2}$ is reminiscent of the formula (in the notation of Silhavy, 2004 , relation (91), p.43)

$$
f(F)=\frac{1}{2} \mu r^{\frac{1}{3}}\left(\alpha_{3}^{2}+\alpha_{2}^{2}+\alpha_{1}^{2} / r\right)
$$

for the energy density for nematic elastomers derived from statistical models of polymers in Bladon et al, 1993, and studied from the point of view of the calculus of variations by DeSimone and Doltzmann, 2002, and by Silhavy, 2004, under the constraint of macroscopic incompressibility $\operatorname{det} F=1$. In (71), $\mu$ is the shear modulus of the material, $r>1$ is the step length anisotropy parameter that describes the average shape of molecular coils, and $\alpha_{1}^{2} \geq \alpha_{2}^{2} \geq \alpha_{3}^{2}$ are the eigenvalues $c_{\max } \geq c_{\text {mid }} \geq c_{\text {min }}$ of $C=F^{T} F$. Therefore, the pure-phase minimization carried out here for the energy $\Psi_{K O}$ in the case of the prolate phase provides a counterpart of an energy density derived via statistical methods for a specific class of polymers. 
We note for later use that for $\nu>1+\sqrt{2}$ the stress $S_{K O}^{p r o}(F, K)$ for $K=$ $-\frac{1}{\nu} I+\frac{\nu+1}{\nu} e_{\min } \otimes e_{\min }$ is given by

$$
S_{K O}^{p r o}\left(F,-\frac{1}{\nu} I+\frac{\nu+1}{\nu} e_{\min } \otimes e_{\min }\right)=\frac{\alpha}{\nu^{2}} F\left(I+\left(\nu^{2}-1\right) e_{\min } \otimes e_{\min }\right) .
$$

As in the case of the oblate phase, the stress in the previous formula need not be completely determined by $F$, although the volume integrals in the right-hand side of the relation (70) are completely determined by the field $F$.

\subsection{Pure-phase energy minimization among the $\Psi_{K O}$-phases}

The formulas (58), (60), (63), (67), and (70) provide the minimum value of the augmented energy for each pure phase of the body. For each non-zero value of the ratio $\nu=\frac{\alpha_{\perp}}{\alpha_{d}}$ we compare in this subsection the minimum values for those phases that may be present for the given $\nu$.

For the interval $-\infty<\nu \leq-1$, the classical, spherical, prolate, and oblate phases all may be present, and comparison of (58), (60), (67), and (70) along with the use of the inequality $\frac{\nu^{2}+3 \nu+1}{\nu^{3}} \leq 1$, that holds throughout the interval $-\infty<\nu \leq-1$, permit us to conclude that the spherical phase provides the minimum augmented energy among all phases available for $-\infty<\nu \leq-1$. (Actually, the augmented energies for all phases are the same when $\nu=-1$.)

For the intervals $-1<\nu<0$ and $0<\nu<1$, the only phase that may be present is the classical phase, so that it provides the minimum augmented energy on these intervals.

The textured phase appears only for $\nu=1$, and (63) and (58) show that the minimum augmented energy in the textured phase agrees with that for the classical phase, and we need not consider the textured phase further in this connection. For $\nu \geq 1$, the only remaining phases to be considered are the classical and prolate phases. The formulas (58) and (70) and the properties of $\frac{P(\nu)}{\nu^{3}}$ noted in the previous subsection imply that the classical phase provides the smaller augmented energy on $1<\nu<1+\sqrt{2}$, while prolate phase provides the smaller augmented energy on $1+\sqrt{2}<\nu$.

In summary, the least augmented energy is provided in the interval $-\infty<$ $\nu<-1$ by the spherical phase, in the intervals $-1 \leq \nu<0$ and $0<\nu \leq 1+\sqrt{2}$ by the classical phase, and in the interval $1+\sqrt{2}<\nu$ by the prolate phase. Therefore, the pure-phase minimization problem for a body with free energy response function $\Psi_{K O}$ selects exactly one among the spherical, classical, and prolate phases in each of the four intervals listed above. Submacroscopically stable equilibria of such a body, if any do exist, must appear among these phases. 


\subsection{Boundary value problems for the determination of submacroscopically stable equilibria}

In order for a solution $(g, K)$ of the pure-phase minimization problem to provide a submacroscopically stable equilibrium configuration, the macroscopic deformation field $g$ and the tensor field $K$ must satisfy the placement boundary condition (17), as well as the equilibrium condition and the traction boundary condition (56) and (57). In this subsection we record the resulting boundary value problem for each of the solutions of the pure-phase minimization problem obtained above. We note at the outset that the case of the spherical and of the classical phase are somewhat simpler, because both $K$ and the stress $S$ are completely determined by the deformation gradient $F=\nabla g$.

For the interval $-\infty<\nu \leq-1$, the discussion in the previous subsection showed that the spherical phase yields the solution of the pure-phase minimization problem. Accordingly, we use the formula (61) to write down the corresponding forms of the equilibrium equation and boundary conditions:

$$
\begin{aligned}
\operatorname{div}\left(\frac{\alpha \backslash}{\nu^{2}} \nabla g\right)+b_{\text {ref }} & =0 & & \\
\left(\frac{\alpha \backslash}{\nu^{2}} \nabla g\right) N & =s_{t} & & \text { on } \partial_{t} B \\
g & =g_{p} & & \text { on } \partial_{p} B .
\end{aligned}
$$

Thus, the macroscopic deformation field $g$ that determines a submacroscopically stable equilibrium configuration for $\nu \leq-1$ is a solution of a mixed boundaryvalue problem for the non-homogeneous Laplace equation.

For the cases $-1<\nu<0$ and $0<\nu \leq 1+\sqrt{2}$, the discussion above shows that the only solution of the pure-phase minimization problem is the classical phase, i.e., $K=I$, and the formula (59) then yields the boundary-value problem:

$$
\begin{aligned}
\operatorname{div}(\alpha \backslash \nabla g)+b_{\text {ref }} & =0 & & \\
(\alpha \backslash \nabla g) N & =s_{t} & & \text { on } \partial_{t} B \\
g & =g_{p} & & \text { on } \partial_{p} B .
\end{aligned}
$$

Formally, this mixed problem for the non-homogeneous Laplace equation in the case of the classical phase is obtained from the one in the case of the spherical phase by replacing $\alpha \backslash / \nu^{2}$ in (73) and (74) by the larger number $\alpha_{\backslash}$.

For the case $1+\sqrt{2}<\nu<\infty$, the solution of the pure-phase minimization problem necessarily has $K=-\frac{1}{\nu} I+\frac{\nu+1}{\nu} e_{\min } \otimes e_{\min }$ and has stress $S$ given by (72). Here, for each $X$ in $B, e_{\min }(X)$ is a unit vector satisfying

$$
C(X) e_{\min }(X)=c_{\min }(X) e_{\min }(X)
$$

with $c_{\min }(X)$ the minimum element in the spectrum of $C(X)=(\nabla g)^{T}(\nabla g)(X)$. If $c_{\min }(X)$ has algebraic multiplicity one, then the $\operatorname{diad} e_{\min }(X) \otimes e_{\min }(X)$ is completely determined by $F(X)$. However, in the most general case, $e_{\text {min }}$ must 
be determined as a unit vector field satisfying (79) as well as the equilibrium equation

$$
\operatorname{div}\left(\frac{\alpha_{\backslash}}{\nu^{2}} \nabla g\left(I+\left(\nu^{2}-1\right) e_{\min } \otimes e_{\min }\right)\right)+b_{r e f}=0
$$

and the boundary conditions

$$
\begin{aligned}
\frac{\alpha_{\backslash}}{\nu^{2}} \nabla g\left(I+\left(\nu^{2}-1\right) e_{\min } \otimes e_{\min }\right) N & =s_{t} & & \text { on } \partial_{t} B \\
g & =g_{p} & & \text { on } \partial_{p} B .
\end{aligned}
$$

The boundary value problem (79)-(82) for the case of the prolate phase is a nonlinear problem, unlike those for the case of the spherical and classical phases. The form of this problem suggests that it may be fruitfully studied with tools and results for implicit partial differential equations (Dacarogna and Marcellini, 1999).

In all three cases discussed in this subsection, the macroscopic deformation $g$ corresponding to a submacroscopically stable equilibrium configuration must not only be a solution of the corresponding boundary-value problem but also must be injective and must be orientation preserving: $\operatorname{det} \nabla g(x)>0$ for all $x$ in $B$.

\section{Example: near-sighted fluids}

At the end of Section 3.3 we provided in relations (34)-(38) the basis for determining partially relaxed equilibrium configurations in the form $(g, K)$. These relations are appropriate for cases where the Helmholtz free energy density assumes a simple form when expressed in terms of the variables $F=\nabla g$ and $K$. In this section, we consider in this vein the example of a near-sighted fluid (Deseri and Owen, 2003) for which the free energy response takes the form

$$
\bar{\Psi}(\nabla g, K)=\psi(\operatorname{det} K) .
$$

with $\psi$ a smooth function on the interval $(0,1]$.

As was the case in the preceding example and in the general discussion in Subsection 3.4, the first step in determining submacroscopically stable equilibria for the near-sighted fluid is that of solving the following minimization problem: for a given macroscopic deformation $g$ that satisfies the placement boundary condition, find the tensor fields $K$ that minimize the augmented energy (38) subject to the relations (35)-(37). For the near-sighted fluid, this problem reduces to the pointwise minimization of the Helmholtz free energy density $\psi(\operatorname{det} K)$ subject to the constraints

$$
\begin{aligned}
\psi^{\prime}(\operatorname{det} K)\left(K^{2}-3 K+I\right) & =0 \\
s k\left(\psi^{\prime}(\operatorname{det} K) F^{-T}\left(K^{-T}-2 I\right) F^{T}\right) & =0 \\
0<\operatorname{det} K & \leq 1 .
\end{aligned}
$$


We note here that for the near-sighted fluid, the stress relation (10) becomes

$$
S=\operatorname{det} K \psi^{\prime}(\operatorname{det} K) F^{-T}\left(I-2 K^{-T}\right)
$$

To carry out the first step described above, we observe that if the Helmholtz free energy response function $\psi$ has a global minimum at a tensor $K$ for which $\operatorname{det} K \in(0,1]$ and $\psi^{\prime}(\operatorname{det} K)=0$, then the constraints $(84)-(86)$ all are satisfied. Moreover, the relation (87) tells us that the stress is zero. Consequently, we call tensors $K$ that minimize $\psi$, that satisfy $\operatorname{det} K \in(0,1]$ and for which $\psi^{\prime}(\operatorname{det} K)=$ 0 stress-free solutions of the first-step minimization problem. Of course, if $K$ is a stress-free solution, then any multiple of $K$ by a tensor $U$ with $\operatorname{det} U=1$ also is a stress-free solution.

Solutions $K$ of the first-step minimization problem that are not stress-free would necessarily satisfy $\psi^{\prime}(\operatorname{det} K) \neq 0$, so that (84) and (85) would become

$$
\begin{aligned}
K^{2}-3 K+I & =0 \\
s k\left(F^{-T}\left(K^{-T}-2 I\right) F^{T}\right) & =0 .
\end{aligned}
$$

It was shown by Deseri and Owen, 2003, that these two equations are equivalent to

$$
\begin{array}{r}
H^{2}-3 H+I=0 \\
s k H=0
\end{array}
$$

where $H:=G F^{-1}=F K F^{-1}$, and the only solutions $H$ of these two equations are

$$
\begin{array}{r}
H_{s p h}:=\left(1-\gamma_{0}\right) I \\
H_{\text {pro }}:=H_{s p h}+\left(1+2 \gamma_{0}\right) d \otimes d .
\end{array}
$$

Here, $d$ is an arbitrary unit vector, and $\gamma_{0}:=\frac{\sqrt{5}-1}{2}$ is the "golden mean." The tensor $H_{s p h}$ is said to determine the spherical phase of the near-sighted fluid, while $H_{\text {pro }}$ is said to determine the prolate phase of the fluid. (The term "elongated phase" was used in place of "prolate phase" in Deseri and Owen, 2003.) In terms of the variable $K$, solutions of the first-step minimization problem that are not stress-free would be among the list

$$
\begin{array}{r}
K_{s p h}:=\left(1-\gamma_{0}\right) I \\
K_{p r o}:=K_{s p h}+\left(1+2 \gamma_{0}\right) F d \otimes F^{-T} d .
\end{array}
$$

We have $\operatorname{det} K_{s p h}=\operatorname{det} H_{s p h}=\left(1-\gamma_{0}\right)^{3}$ and $\operatorname{det} K_{\text {pro }}=\operatorname{det} H_{\text {pro }}=1-\gamma_{0}$, and we may conclude that a solution $K$ of the first-step minimization problem that is not stress-free determines the spherical or the prolate phase of the body, has $\operatorname{det} K=\left(1-\gamma_{0}\right)^{3}$ or $\operatorname{det} K=1-\gamma_{0}$, and satisfies $\psi^{\prime}(\operatorname{det} K) \neq 0$. Because both $1-\gamma_{0}$ and $\left(1-\gamma_{0}\right)^{3}$ are interior points of the domain of the smooth function $\psi$, each of these numbers can deliver the minimum value of $\psi$ only if $\psi^{\prime}$ vanishes at 
that number. We conclude that every solution $K$ of the first-step minimization problem is stress-free.

The conclusions reached above for the first-step minimization problem greatly simplify the second step of the procedure for determining submacroscopically stable equilibria. In fact, necessary conditions for the existence of a stressfree equilibrium configuration $(g, K)$ are the vanishing both of the body force field $b_{r e f}$ and the applied surface traction field $s_{t}$. Because the only remaining restriction on a candidate $(g, K)$ for a submacroscopically stable equilibrium configuration is the placement boundary condition, we obtain the following characterization of the submacroscopically stable equilibria of the near-sighted elastic fluid: given the vanishing of the body force field and the applied surface traction field, a structured deformation $(g, K)$ is a submacroscopically stable equilibrium configuration of the near-sighted fluid if and only if $g$ satisfies the placement boundary condition and, at each point $X$ in $B$, $\operatorname{det} K(X)$ minimizes $\psi$ and satisfies $\psi^{\prime}(\operatorname{det} K(X))=0$. We note that, for a submacroscopically stable equilibrium configuration $(g, K)$, if the body $B$ is a connected set, then the continuity of the field $K$ on $B$ implies that the scalar field $\operatorname{det} K$ is constant or takes values throughout a non-trivial interval on which $\psi^{\prime}$ vanishes.

\section{Conclusions and outlook}

The notion of a submacroscopically stable equilibrium configuration of a body and the procedure introduced here for the determination of submacroscopically stable equilibria provide the basis for selecting in a systematic way preferred submacroscopic geometrical states of bodies in equilibrium. The augmented energy underlies this methodology and provides not only a functional of $g$ and $\Delta=G-M$ that is stationary for fixed $\Delta$ at equilibrium configurations but also that cannot increase under purely submacroscopic, quasistatic processes in time-independent environments.

These ideas were developed in Section 3 for arbitrary elastic bodies undergoing disarrangements and dissipation and were illustrated for specific bodies in Sections 4 and 5. In particular, the bodies studied in Section 4 have biquadratic free energy response, and their submacroscopically stable equilibria arise only for submacroscopic geometries associated with the spherical phase, the classical phase, or the prolate phase, depending upon the value of the ratio $\nu$ of the two response moduli $\alpha \backslash$ and $\alpha_{d}$. For a specific range of the parameter $\nu$, the formula (70) obtained in Section 4 for the minimum Helmholtz free energy density within the prolate phase is reminiscent of the formula (71) obtained in [?] by a different method, one based on a bottom-up, statistical calculation. The nearsighted fluids discussed in Section 5 possess universal spherical and universal prolate phases (Deseri and Owen, 2003) that generally are not stress-free, but the submacroscopically stable equilibria that are available to such fluids all are stress-free.

Boundary-value problems for the macroscopic deformation were recorded for all of the submacroscopically stable equilibria identified in Section 4. For those 
corresponding to the spherical and classical phases, the boundary-value problems for the macroscopic deformation are mixed, linear problems for the nonhomogeneous Laplace's equation. For those corresponding to the prolate phase, non-linear problems arise whose form suggests the use of techniques available in the literature (Dacarogna and Marcellini, 1999). When the boundary-value problem for macroscopic deformation has a classical, smooth solution $g$ that is injective and orientation preserving, then the structured deformation $(g, \Delta)$ would provide a submacroscopically stable equilibrium configuration of the body in the present sense. Alternatively, when only solutions with less smoothness emerge, the boundary-value problem would not provide submacroscopically stable equilibria in the present sense. At the same time, the existence of solutions with less regularity would indicate that a more general notion of submacroscopically stable equilibria is required, one that might admit a larger class of macroscopic deformations that support additional geometrical structures such as those associated with mixtures of phases.

The inclusion of mixtures of phases could also be undertaken at an earlier point in this development by relaxing at the outset the requirement that $F$ and $G$ be smooth fields, with the result that the solutions of the relations (35)-(37) could allow the body to be in different phases at different points. However, the possible presence of coherent phase boundaries in the body suggests that the definition (21) of the augmented energy be modified to include a contribution from the phase boundaries. Moreover, such phase boundaries would be restricted by the condition that the traction field be continuous, a consequence of balance of forces for the continuum.

The present theory can be further enriched in order to include internally constrained materials. A typical macroscopic internal constraint is embodied in the condition $\operatorname{det} F=1$, that of macroscopic incompressibility. An example of a submacroscopic internal constraint is one characterized by the condition $\operatorname{det} F=\operatorname{det} G$ or, equivalently, $\operatorname{det} K=1$ that prevents disarrangements from causing a change in volume. A description of such constrained materials in the setting of elastic bodies undergoing disarrangements and dissipation (Deseri and Owen, 2003) would require the introduction of reaction stresses that are not constitutively determined, e.g., not arising as partial derivatives of the free energy response function (or in any other way determined by that response function). These reaction stresses would enter into many of the field equations employed in the present article and, generally, would lead to a broader class of equilibrium configurations of the body.

\section{Acknowledgement}

The authors thank the Center for Nonlinear Analysis (NSF Grant No. DMS0405343) in the Department of Mathematical Sciences, Carnegie Mellon University, for its support during the preparation of this paper. L. Deseri also wishes to acknowledge support under the Italian grant PRIN 2005 "Modelling and Approximation Techniques in Advanced Problems in the Mechanics of Materials and Structures" from the M.U.R. In addition, D. R. Owen acknowledges the support provided to N. Kirby by the SURF program at Carnegie Mellon and by 
the US National Science Foundation, Division of Mathematical Sciences, Award 0102477 .

\section{References}

Bladon, P, Tarentiev, E. M., Warner, M., 1993. Transitions and instabilities in liquid-crystal elastomers.Phys. Rev., E. 47, R3838-R3940.

Dacarogna, B. Marcellini, P., 1999. Implicit Partial Differential Equations. Birkhäuser, Boston, etc.

Del Piero, G. Owen, D.R., 1993. Structured deformations of continua. Arch. Rational Mech. Anal. 124, 99-155.

Del Piero, G. and Owen, D. R., 1995. Integral-gradient formulae for structured deformations. Arch. Rational Mech. Anal. 131, 121-138.

Deseri, L. and Owen, D. R., 2000. Active slip-band separation and the energetics of slip in single crystals. Int. J. Plasticity. 16, 1411-1418.

Deseri, L. and Owen, D. R., 2002a. Energetics of two-level shears and hardening of single crystals. Math. Mech. Solids.7, 113-147.

Deseri, L. and Owen, D.R., 2002b. Invertible structured deformations and the geometry of multiple slip in single crystals, Int. J. Plasticity. 18, 833-849.

Deseri, L. and Owen, D. R., 2003. Toward a field theory for elastic bodies undergoing disarrangements. J. Elasticity 70, 197-236.

DeSimone, A., Doltzmann, G., 2002. Macroscopic response of nematic elastomers via relaxation of a class of SO3-invariant energies. Arch. Rational Mech. Analysis. 161, 181-204.

Kirby, N. and Owen, D. R., 2005. Unpublished communication.

Silhavy, M., 1997 The Mechanics and Thermodynamics of Continuous Media. Springer, Berlin.

Silhavy, M., 2004. Energy Minimization for Isotropic Nonlinear Elastic Bodies, in Multiscale Modeling in Continuum Mechanics and Structured Deformations. Springer, Wien NewYork.

Truesdell, C. and Noll, W., 2002. The Non-Linear Field Theories of Mechanics, 2nd edn. Springer-Verlag, Berlin. 